\title{
Los retos de la era de las TICs: nativos digitales contra inmigrantes
}

\section{The Challenges of the TICs' Age: Digital Natives Versus Inmigrants}

\author{
David Caldevilla Domínguez \\ Universidad Complutense de Madrid \\ davidcaldevilla@ccinf.ucm.es
}

\begin{abstract}
Resumen
Los "Nativos Digitales" tal y como los bautizó Prensky en 2001 son una nueva generación que supone un desafío para las nuevas líneas de investigación en semiótica dado su nuevo lenguaje y el desarrollo de las plataformas 2.0. Este grupo de jóvenes nacidos a finales de la década de los 80 piensan, actúan, se relacionan e incluso estudian de manera digital planteando nuevos retos para los investigadores.

En este artículo se analizan las características que han llevado a separar de forma precisa a estos "nativos digitales" de los inmigrantes forzosos desde la era analógica y que deben adaptarse a las vicisitudes de la Red de Redes para mantenerse vivos en el mundo actual cada vez más interconectado.
\end{abstract}

Palabras clave: nativos digitales, semiótica, aprendizaje, edades mediáticas.

\begin{abstract}
The "Digital Natives" as Prensky baptized in 2001 are a new generation that is a challenge for the new lines of research in semiotics as its new language and development platform 2.0. This group of young people born in the late 80 s think, act, relate and even study in a digitally way, posing new challenges for researchers.

This article discusses the features that have led to separate precisely these "digital natives" of forced migrants from the analog era that must adapt to the vicissitudes of the World Wide Web to stay alive in today's world increasingly interconnected.
\end{abstract}

Keywords: Digital natives, semiotics, learning, media ages. 


\section{La importancia de la semiología en el estudio de los nuevos procesos de interacción comunicacional}

Nos encontramos en una época que podemos titular de comunicacional. Imagen, sonido, música, videos, textos... se (re)producen a diario y se comparten rápidamente de un lugar a otro del mundo gracias a las nuevas Tecnologías de la Información del Siglo XXI.

Esta virtualización de la comunicación no pasa desapercibida. Comunidades en línea, redes sociales 2.0, las wikis, las bitácoras, los fotologs, los chats, las videoconferencias, los hipervínculos, los hipertextos, las creaciones colectivas e, incluso los mundos virtuales, han convergido en un mundo enteramente tecnológico que avanza a una velocidad vertiginosa.

A esto se suma la convergencia, que antes de ser mediática o tecnológica, fue cultural. La cultura participativa, la acción colectiva, la democratización de los ordenadores fueron el caldo de cultivo para este nuevo universo en red. Así, las computadoras pasaron de ser meras herramientas de trabajo, que conllevaban un desembolso económico importante y que estaban sólo al alcance de empresas y más tarde de la élite económica de particulares, se convirtieron en un elemento común y cotidiano que hoy ya se utiliza no tanto para trabajar sino para conformar nuestro ocio y proveernos de información, labor ésta en la que dan alcance a la hasta ahora omnímoda televisión.

Los semióticos afirmaron que todo era signo. Hoy, habiendo heredado las teorías de la semiosis infinita, podemos corroborar empíricamente la multitud de formas de acceder a los signos, a producirlos, a crearlos y sobre todo a compartirlos mediante modelos de comunicación y visualización en constante evolución. ¿Expresión o explosión semiótica?

Es conveniente, por tanto, reinterpretar ciertos conceptos para un mejor posicionamiento en este presente virtual y comprender qué supone haber nacido, crecido o simplemente haberse adherido a la Era Digital.

En el bosque de signos en el que vivimos (como bien representó Baudelaire), tal como apostilla Peirce (1994), todo es semiosis infinita. Hjmeslev (1971) alude al afianzamiento de la humanidad por medio del lenguaje, sacando a la palestra la teoría de la enunciación de Benveniste (1977): "La existencia misma del hombre está estructurada por la lengua. La lengua es la única forma en que podemos revestir al mundo. No podemos concebir ninguna otra forma de la existencia que la que es dada por la lengua”.

Lotman (1999) llegará más allá declarando 'la cultura como lenguaje'. Abordando el tema de forma silogística. Si partimos de la premisa de que el lenguaje es signo y los hombres somos lenguaje, la humanidad se construye en base a su afianzamiento sígnico. Es decir, somos signos. Pero yendo un poco más allá: si la cultura es lenguaje y comunicación, también ésta será conformada por una red de signos, lo que lleva a considerar a la cultura como un mecanismo metasígnico y plurilingüe.

Tal como explica Bougnoux (1999): "El hombre desciende más del signo que del mono (en francés, signe)” y continúa afirmando que "el imperio de los signos duplica el mundo natural, la semiosfera (que integra la cultura en general) contiene a la biosfera (la naturaleza, el mundo animal, vegetal, etc.)”. En un tour de force último, derivaremos que la tecnología desborda los espacios (iy tiempos!) para que vivamos las vidas que queremos. 
Desde la definición clásica, "el signo es una cosa puesta en el lugar de otra cosa”, (alien et alter), Saussure vinculaba un significante y un significado, en su ambivalencia inseparable. Fue Hjelmslev quien propuso el significante como plano de la expresión y el significado como plano del contenido planteando así la analogía de la lengua refiriéndose a una lingüística estructural en la teoría de la información, adicionándoles los conceptos de mensaje y código.

La tecnología ha cambiado la cultura y por ende la forma de activar y desactivar los mensajes y sus actantes. ¿En qué aspectos podemos incardinar el 'nuevo acto comunicativo' tamizado por la tecnología. A este debate se han apuntado muchos estudiosos. Quizás coincidimos con el análisis de Jorge Lozano, quien retomará el concepto al afirmar que "el acto comunicativo no es transmisión pasiva de información, sino una traducción, una recodificación del mensaje” y agregará "en la base de las relaciones humanas se encuentra el acto de comunicación, acto que debe ser considerado como un intercambio de cosas que se equivalen”. Permiten, por lo tanto, situar a las personas entonces, en el marco de una cultura de signos, de enlaces, de hipertextos, o de links.

¿Estamos ante una revolución? Sí y no. Una revolución conlleva mudanza radical de formas y estructuras y en ese aspecto no hay duda de que la comunicación del siglo XXI poco o nada tendrá que ver con las anteriores. Esto es indudable, pero, por el contrario, el actor de la comunicación y sus contenidos (allende las formas) siempre será el ser humano y su naturaleza como centro de las cosas. Jorge Hidalgo Toledo (1995) señala que "Una nueva revolución simbólica/antropológica se está viviendo; los resultados: cambios profundos en la personalidad, nuevos modos de interacción, diálogo y significación. Necesariamente toda nueva sintaxis establece una nueva semántica y por ende, una nueva pragmática.”

Estas reflexiones de Hidalgo hacia los nuevos modos de interacción o diálogo nos abren la puerta teórica a las nuevas tecnologías digitales como los vehículos de comunicación semiótica del siglo XXI. La parte técnica, que posibilita un tipo u otro de comunicación y mensajes se debe a la prolífica mente de los ingenieros, pero esto, como dría Kipling, 'es otra historia'.

\section{La temporalidad del hombre como vehículo para los nuevos cambios generacionales}

El hombre es temporal y su lucha contra Kronos es una guerra con muchas batallas perdidas. El ansia de la inmortalidad ha empujado a muchos creadores hacia la poética senso lato.

Existen dos formas de tiempo que conviene distinguir: el tiempo histórico y el tiempo personal o biográfico. El tiempo es continuo y perpetuo pero está segmentado. Podemos hablar de eras, épocas y generaciones por ejemplo.

Algo parecido ocurre en la vida: la existencia también es un continuum, desde el nacimiento hasta la muerte, pero está articulada en edades. El problema radica en que la edad se ha contemplado desde el prisma biológico resumiéndola en diferentes fases como pueden ser el nacimiento, la niñez, la juventud, la madurez, la vejez y por último la muerte. Sin embargo, una 
visión más amplia de lo que conlleva el concepto de edad nos conduce a un estadio bastante más complejo.

Las edades entendidas como fases no son estáticas, no son científicas (como la ciencia y su método intentan demostrar), están muy relacionadas con lo vivido, lo aprendido, lo experimentado e incluso lo padecido o lo observado. Por lo tanto podemos colegir que las edades también son, entre otras cosas, cognitivas y perceptivas (en formas y contenidos). El problema radica en que ha predominado el punto de vista orgánico; se ha visto las limitaciones de cada edad como un problema de desarrollo biológico en diferentes fases y que han sido tildadas de diferentes maneras, desde el nacimiento hasta la vejez y finalmente la muerte.

Desde el punto de vista cognitivo, la edad es un proceso de adquisición de conocimientos a lo largo de un tiempo determinado que nos posibilitan el acceso a ciertos saberes básicos para el desarrollo cultural o intelectual del individuo.

Las edades mediatizan el nivel óptimo de aprendizaje de nuevas tareas. La tecnología, forma parte cada vez más de nuestra vida, y por tanto, los conocimientos adquiridos en ese sentido, forman parte de una cultura digital común, globalizada. Para Prensky la generación de jóvenes nacidos a finales de la década de los ochenta (a los que ha bautizado como "nativos digitales") acepta las nuevas Tecnologías de la Información y Comunicación (TICs) como algo casi innato, asumido y practicado desde que sus componentes son niños. ¿Pero esto es así para el resto de personas? Supone una necesidad imperiosa hoy en día adherirse a esta corriente digital, en especial a sus conocimientos "analógicos", convirtiéndose así sus neófitos en inmigrantes digitales (aquellos que han emigrado del analógico al digital dado que no pertenecen a este país imaginario de 'nativos digitales').

Es por ello por lo que necesitamos hacer una serie de apostillas sobre el término digital y analizar cómo nos influye personalmente la tecnología tanto a aquellos que han tenido que adaptarse a ella como a esa nueva generación emergente de jóvenes con unos rasgos característicos muy pronunciados y que formarán parte de la sociedad futura desde su cuna.

\section{Nativos digitales, definición y características}

Ya hemos comprobado como un investigador de referencia es Marc Prensky (2001) en su ensayo titulado La muerte del mando y del control, en el que define a los nativos digitales como aquellas personas que han crecido con la red, es decir, nacieron en un mundo ya digital donde las nuevas tecnologías se desarrollaban rápidamente, y los distinguía de los inmigrantes digitales llegados más tarde a las TICs.

Pero no sólo el estadounidense Prensky ha iniciado el debate sobre lo nativo e inmigrado digital. Voces como la de Boschma (2008) retoma este análisis unos años más tarde y pone nombre a esta nueva generación de jóvenes nacidos en entornos desarrollados tecnológicamente. Los denominó 'generación Einstein' en honor al científico por antonomasia del siglo XX. 
Boschma puntualiza que las personas que habían nacido después de 1988 forman parte de una generación de nativos digitales que "les ha dotado de una manera de procesar la información más cercana a Einstein (creativo y multidisciplinar) que a Newton (racional, lógico y lineal)”. En nuestra opinión, quizá sea debida esta puntualización a la superación de la lógica aristotélica por la booleana y mucho tenga que ver con este concepto el análisis de la tecnología y del usuario digital que efectúa Boschma, que se nos presenta como más pluridisciplinar.

Sin embargo, una de las carencias de estas definiciones es que sólo se refieren a un contexto tecno-cultural (digital) vinculado al nacimiento de usuario, pasando por alto otras características o variables, como pueden ser su generación precedente y sus vínculos con los demás usuarios y con las máquinas cada vez más complejas y programas cada vez más accesibles.

Por ejemplo, según esta definición, el nativo digital ostenta esa condición simplemente por el mero hecho de haber nacido en una determinada década rodeado de un contexto que podemos llamar "tecnocultural digital" dejando fuera otros aspectos sociodemográficos que describen esta brecha generacional.

Nativo digital es definido como aquella persona que ha nacido y ha crecido en un entorno digital; sin embargo no está de más reconocer que existen jóvenes que a pesar de haber nacido en las mismas circunstancias, no son "digitales” y lo que es peor, difícilmente accederán a serlo.

Comúnmente se define al "nativo digital" como la "persona que ha crecido rodeado de tecnología digital como ordenadores, Internet, móviles y MP3”. Esta definición también cae en el error de incidir en los elementos con qué ha crecido esta generación, no con quién. Palabras que recoge el investigador Juan Freire en su bitácora. La edad, la pertenencia a una generación cronológica, es un factor necesario pero no suficiente. Tampoco se identifican las razones últimas de la pertenencia de una persona a esta categoría aludiendo a criterios objetivables:

Not all people born during a certain period of history (say, after the advent of BBSes) are Digital Natives. Not everyone born today lives a life that is digital in every, or indeed any, way. For starters, only about 1 billion of the 6.7 billion people in the world have regular access to the supposedly "World Wide Web." In other cases, young people we are meeting choose to have little to do with digital life. (Palfrey, cit. por Freire, 2007)

Más de acuerdo nos hallamos con Juan Freire cuando asevera que ni el contexto digital, ni la edad, delimitan por sí solos la llamada "tecnoculturalidad”. Como dice John Palfrey, director del prestigioso Berkman Center of Internet and Society en su bitácora, "hay nativos que no son digitales e inmigrantes digitales con comportamientos digitales envidiables para un nativo”.

Debemos por lo tanto continuar buscando las características que nos lleven a designar con precisión y exactitud quiénes son los nativos digitales y por qué podemos considerarlos así, y quiénes meros inmigrantes digitales adaptados (con mayor o menor fortuna) a la situación digital por distintas circunstancias como son: las obligaciones laborales, la necesidad de estar informados, el entretenimiento... No es la fecha de nacimiento, ni el contexto digital, sino el 
contexto vincular (con los otros y con las máquinas) lo que determinará la "natividad digital” tal y como asegura Balaguer. ${ }^{1}$

Por eso, la natividad digital no pasa solamente por la fecha de nacimiento ni por pertenecer a una generación concreta. Lo que verdaderamente define a un nativo digital es su simbiosis con las máquinas y nuevas formas de comunicación interactiva (tal y como señalan De Kerkhove y Turkle). En esta línea de trabajo, Skiba y Barton (Adapting your teaching to acommodate the net generation of learners, 2006) puntualizan las características de esta generación, nacida después de 1980, incluyendo: competencias digitales, aprendizaje experiencial y activo; interactividad y colaboración; inmediatez y conectividad.

Nos hallamos ante un verdadero 'Usted está aquî' de la comunicación tecnologizada. Prensky en 2004 identifica 18 características en las que los nativos digitales son diferentes al resto de generaciones:

1. Se comunican de forma diferente con los demás inventando su propio lenguaje adaptado a la velocidad del teclado del ordenador y el móvil, prefieren los medios asincrónicos y no necesitan conocer a sus interlocutores para mantener una conversación con ellos. Se identifican mediante apodos y avatares. En este punto los semiólogos tienen mucho campo de análisis y por él apostamos en futuras investigaciones.

2. Comparten información, fotografías, vídeos, textos, etc., de manera diferente aprovechando al máximo las posibilidades que les ofrecen las $2.0 \mathrm{y}$ los sistemas de intercambio P2P (persona a persona). Los derechos de autor en estos momentos limitan este tipo de información al poner en común creaciones protegidas por sus autores.

3. Realizan sus transacciones económicas (compras y ventas) de forma diferente teniendo la red como mercado sin limitación espacio-temporal y están habituados a comprar y vender a través de sistemas de subasta (como el famoso e-Bay). Las pasarelas de seguridad de las financieras son punteras en la encriptación de este tipo de mensajes continentes de datos confidenciales.

4. Intercambian impresiones de manera diferente (especialmente volcados al intercambio de contenidos de todo tipo). La censura de los Estados está poniendo en la palestra los límites de unos y otros.

5. Crean de manera diferente y están habituados a la cultura del remix y del famoso "cortapega” mezclando y fusionando todo tipo de contenidos de diversos autores y de formatos. La creación de los ususarios hace que los papeles de receptor-emisor sean reversibles por primera vez en la historia de manera eficaz.

6. Se citan entre ellos de manera diferente (empleando las redes sociales, la mensajería informática y los mensajes a dispositivos móviles). Son los mentores de los flashmobs (multitudes instantáneas), es decir, acciones organizadas en las que un gran número de jóvenes (o no) que no

\footnotetext{
1 Roberto Balaguer Prestes Psicólogo egresado de la Universidad de la República (Uruguay). Postgraduado en Psicoterapia Psicoanalítica de Niños y Adolescentes y de Adultos. Coordinador de Grupos de Trabajo en los tres Congresos Online del Observatorio para la Cibersociedad, con sede en Barcelona http://www.robertobalaguer.com
} 
tienen por qué conocerse entre sí se reúnen en un lugar público para realizar algo inusual (con fines de entretenimiento o incluso fines políticos) y luego se dispersa. Las nuevas relaciones sociales se escriben con 0 y 1 creando sociedades virtuales.

7. Coleccionan de manera diferente (no juntan los típicos sellos, cromos, latas de cerveza... utilizan los medios para etiquetar, organizar, juntar, clasificar y compartir en la rápidamente, todo tipo de hipervínculo). Los usos de los nuevos tiempos varían con los formatos y posibilidades. De hecho el espacio de las casas, cada vez más reducido, invita al almacenamiento digital. No se coleccionan cosas sino sus representaciones.

8. Se coordinan de manera diferente. La velocidad de transmisión ayuda a la instantaneidad, otrora imposible.

9. Evalúan de forma diferente dándole muchísima importancia a la construcción de la reputación en línea basándose en las opiniones y en el número de visitas a los portales de Internet. La comunicación en medios tradicionales tiene que mudar hacia posturas más flexibles. El modelo de negocio comunicativo que conocíamos hasta hoy ha muerto.

10. Juegan de manera diferente a través de videojuegos en línea donde pueden pasar horas participando en torneos y equipos formados por jugadores en todos los puntos del planeta desconocidos entre sí. El ocio es digital. Se prevén problemas de socialización y patologías nuevas derivadas de ello.

11. Aprenden de manera diferente (ya que sus referentes están en la red), utilizan las wikis y las experiencias y tutoriales realizados por otros usuarios para formarse en lo que necesitan. La eeducación socializa la figura del emisor pero surge el problema más grave de las TICs: iquién controla al maestro?

12. Buscan de manera diferente utilizando los grandes buscadores y siendo muy meticulosos con los tags o palabras clave. Las tecnologías han acabado con la dictadura de la élite cultural. Hoy las fuentes son infinitas pero en detrimento de su calidad.

13. Analizan de forma diferente: Son más críticos y analizan de forma más científica las diferentes situaciones. El engaño y el silencio hoy son imposibles. Como dijera Abraham Lincoln "Se puede engañar a algunos todo el tiempo y a todos algún tiempo, pero no se puede engañar a todos todo el tiempo”. Internet lo hace más posible.

Si esta situación se lleva al límite nos encontramos un panorama clasista en el cual sólo aquellos que disfruten de las ventajas de la red pueden aprovechar las oportunidades que ella ofrece, una cybersociedad en la que los no iniciados quedan fuera. Da miedo, pero no hay por qué preocuparse... de momento. Gracias a Internet el mundo pude saber la opinión de los habitantes de Sarajevo, cuando estaban aislados, en pleno auge de la guerra de los Balcanes, o de los estudiantes chinos (muy moderada, por si acaso) en el aniversario de la matanza de estudiantes de hace años. Hace tiempo que los gurús de la nueva era afirman que Internet es un bien necesario para todos: toda la sociedad debe tener acceso a ella; otra cosa es la cantidad o los objetivos de su uso. (Melero 2000) 
14. Informan de sus opiniones o vivencias de manera diferente utilizando diversos sistemas de microblogging como Twitter o redes sociales. El lector in fábula de Ecco ha pasado a convertirse en lector fabulante y este proceso es irreversible.

15. Programan sus actividades diarias de forma diferente. Gracias al ahorro de tiempo que conlleva el uso de estas tecnologías.

16. Se relacionan con la sociedad de forma diferente teniendo a la red como punto de encuentro con su entorno social más próximo. Las relaciones humanas cambian de estructura, en las que el espacio no es un factor negativo. No obstante hay varios estudiosos que ya hablan de patologías propias del uso y abuso de la red y de las disfunciones sociales de conducta que pueden conllevar.

17. Evolucionan de manera diferente asumiendo papeles y falsas identidades para así poder navegar más fácilmente (con los peligros que aquello conlleva). Los Estados tienden a limitar y limitarán cada vez más estos extremos.

18. Crecen de manera distinta, en muchos casos sin supervisión de los adultos, explorando, buscando, probando, remezclando ideas y transgrediendo reglas (la piratería por ejemplo). La protección de la infancia es una asignatura aún pendiente en la red. No obstante, el lado positivo se deriva del múltiple acceso a fuentes que permiten ampliar horizontes y evitar la manipulación de un Gran Hermano como otrora sucediera.

Surge así una nueva forma de afrontar lo cotidiano:

el nativo digital es multimedia, prefiere el universo gráfico al textual, la hipertextualidad a la linealidad, aprenden en red y de la red, actuando ésta como elemento vertebrador de la socialización, dominan los medios de producción digital y, sobre todo, entienden el mundo como terreno de juego. (Rosel, 2008)

\section{Del "baby boom" 1.0 a la Net-Generation 2.0}

¿Cantidad o calidad? Esta eterna cuestión, sociológicamente planteada, tiene visos de morir sin respuesta. En Growing up digital: The rise of the Net Generation (Dan Tapscott, 2001) se realiza un profundo estudio de las implicaciones económicas y socioeducativas de la aparición de la nuevas tecnologías digitales de información y comunicación. Utiliza los términos de "Net Generation", "N-Generation" o "N-Geners" para referirse a "todos los niños, adolescentes y jóvenes que tienen en 1999 entre 2 y 22 años".

Para justificar este proyecto, el autor se basa en datos que atesoran una imparable generalización del uso de Internet, (al menos en los países más desarrollados) (Tapscott: 2001, 3): según el último estudio Mediascope 2010 sobre el uso de las nuevas tecnologías, la mayoría de adolescentes declara haber utilizado un ordenador, la práctica totalidad en el caso de los videojuegos; incluso aquellos que no tienen acceso a estas tecnologías tienen la capacidad de trabajar con un ordenador con destreza; las nuevas tecnologías digitales tienen para los más jóvenes un atractivo muy importante; la Net-Generation no tiene problemas para entender el 
funcionamiento de los ordenadores o de los programas que éstos utilizan y conservan una actitud proactiva en un medio en el que se sienten actores principales; también utilizan estas herramientas tecnológicas para comunicarse de forma natural, sin distinguir en muchos casos o establecer barreras entre los espacios físico y virtual.

Tapscott parte de la premisa de que existe una dicotomía generacional muy diferenciada, cuyas formas de vida, actitudes y valores se han modelado en circunstancias temporales muy particulares. Los conformantes de la N-Generation, nacidos entre 1977 y 1997, representan un $30 \%$ de la población y son los primeros en superar en número a la generación llamada baby boom: los nacidos entre 1946 y 1964, los, que representan un 29\% de la población (Tapscott: 2001, 15). Así, frente a este grupo de población, nativos de la era digital, se encuentra la generación de sus padres (actuales inmigrantes digitales), cuyo desarrollo estuvo marcado por la llegada de la televisión. En la década de los sesenta, la radio fue sustituida por la pequeña pantalla en los hogares impactando enormemente a los niños y adolescentes de la época. Incluso la disposición del mobiliario de las viviendas cambió adaptándose a este nuevo electrodoméstico con un poder insuperable. La aparición por televisión de imágenes (muy cruentas) sobre la guerra de Vietnam o la llegada del hombre a la Luna, por ejemplo, marcaron su identidad y sus valores.

Del mismo modo que sus progenitores asumieron y entendieron las claves de la sociedad de los medios de comunicación para masas, sus hijos no han tenido problema en adaptarse a un modelo comunicativo donde desarrollan un papel mucho más activo.

Y no olvidemos que Internet sigue brindando una parte muy ínfima de sus posibilidades para que sigamos creciendo en todas las esferas humanas. Crezcamos. La naturaleza de las cosas, también de la Red, nos impone cambios y mejores perspectivas. La medida ha de ser el obtener beneficios aprovechando todo lo que tenemos al alcance desde una visión ética.

Las organizaciones profesionales y las tecnologías de la información como nuevos valores de los Medios de Comunicación de Masas. (Frutos, 2009)

A través de Internet, obtenemos un sinfín de posibilidades de carácter informativo y comunicacional: podemos tener al alcance todo tipo de información, se puede jugar, relacionarse con gente de todo el mundo, etc. Este cambio entre un modelo de comunicación 1.0, es decir, donde el televidente es mero espectador de unas imágenes impuestas sin ningún poder de decisión, a un modelo mucho más interactivo que podemos llamar 2.0, de participación y colaboración, que pronto acabará absorbiendo al primero según la teoría de unificación de pantallas, es la razón por la que los nativos digitales se consideran como la evolución desde la generación de sus padres a un mundo más abierto y plural. Estas técnicas son usadas por los $\mathrm{N}$ Geners para acciones que nos son familiares: educar(se), jugar, comunicar(se), relacionarse... Con Internet tienen acceso a experiencias muy variadas y en numerosas ocasiones resulta muy complicado instaurar un control parental o educativo para impedir que los menores accedan a contenidos inadecuados o que se expongan a situaciones embarazosas, de persecución o acoso.

Las diferencias en la forma de aprender y trabajar de nativos e inmigrantes digitales son latentes. Mientras que los nativos tienen conocimientos avanzados de búsqueda y análisis de 
información, los inmigrantes dejan la información en segundo plano. Otro aspecto por destacar es la función "multitarea". Los nativos digitales están acostumbrados a compaginar varias acciones a la vez (ver la televisión a la vez que navegar por Internet por ejemplo) mientras que los inmigrantes prefieren abarcar menos tareas en conjunto. Los inmigrantes digitales están acostumbrados a trabajar individualmente mientras que los nativos son dados a trabajar en red y en grupo compartiendo sus conocimientos con el resto mediante wikis y foros.

Sirva la siguiente tabla de elaboración propia como resumen de las diferencias entre estas dos generaciones:

Tabla 1. Comparación de actitudes entre nativos e inmigrantes digitales (fuente: elaboración propia)

\begin{tabular}{|c|c|}
\hline Nativos & Inmigrantes \\
\hline Reciben información de manera más rápida. & $\begin{array}{l}\text { Tienen poco aprecio por la información a } \\
\text { adquirir. }\end{array}$ \\
\hline Profieren lo oréfice antos gue ol toyto & Los procesos son individuales. \\
\hline $\begin{array}{l}\text { Defienden el acceso a los contenidos de forma } \\
\text { aleatoria (contenidos). }\end{array}$ & $\begin{array}{l}\text { Prefieren aprender de forma individual sin } \\
\text { simultanear procesos. }\end{array}$ \\
\hline $\begin{array}{l}\text { Funcionan mejor cuando trabajan en red } \\
\text { compartiendo conocimientos. }\end{array}$ & $\begin{array}{l}\text { No creen que los nativos puedan aprender con } \\
\text { éxito mientras ven la TV o escuchan música. }\end{array}$ \\
\hline Prosperan con satisfacción inmediata. & $\begin{array}{l}\text { Tienen poca paciencia para las teleconferencias, } \\
\text { la lógica...etc. }\end{array}$ \\
\hline
\end{tabular}

Vemos cómo la mezcla de estas dos estirpes es posible, pero los nativos presentan un área más amplia de desarrollo.

\section{La alfabetización digital de los nativos}

El concepto de analfabetismo y por necesidad, de alfabetización (ya que son complementarios indefectiblemente) ha variado copernicanamente en los últimos tiempos. La cultura no es sólo impresa puesto que la audiovisual televisiva (incluyendo el soporte VHS, CD, DVD) ha sido tenida como de simple apoyo (reportajes, documentales...). Ahora, el manejo de las herramientas tecnológicas genera unas necesidades educativas totalmente novedosas y unos discentes de perfil curricular distinto al que ha existido hasta el presente.

Podemos decir, tal y como señala María das Graças Targino (2008) que:

En los últimos años se viene produciendo un proceso de reconexión de los ciudadanos, así como un redescubrimiento de la comunidad, con el surgimiento de nuevas comunidades, en muchos casos sin una base geográfica. En este fenómeno los nuevos medios electrónicos y las posibilidades de comunicación online cumplen un papel muy importante, permitiendo la conversión de simples 
grupos de interés en nuevos movimientos sociales y generando nuevos tipos de sociedades en red, interconectadas y activas.

Esto tiene como consecuencia la necesidad de una alfabetización digital, la cual contemplaremos desde dos puntos de vista. Por un lado, desde el prisma de los conocimientos adquiridos gracias a la red por los nativos digitales y sus métodos de aprendizaje y por otro lado, la necesidad de una supervisión de ese aprendizaje por parte de los padres con el fin de evitar que ciertos contenidos inadecuados lleguen a manos de los nativos pudiendo provocar situaciones desagradables.

Los nativos digitales utilizan habitualmente una multitud de servicios que les permiten no sólo comunicarse sino también experimentar sobre su propia identidad y sobre cómo se muestran ante los demás: perfiles redes sociales, bitácoras, espacios donde compartir fotografías, mundos virtuales en donde pueden disponer de un avatar que les represente... Buckinham (2007) realizó una tesis de la correlación creada entre los jóvenes y los medios digitales mediante el concepto de identidad, que tiene tanto un matiz psicológico, en el que destaca la creación de una identidad propia en las fases de la infancia y adolescencia, como un matiz más sociológico, basado en el proceso de socialización. Las nuevas tecnologías procuran un ambiente en donde los jóvenes estudian cómo relacionarse y comunicarse, siendo una parte importante en la construcción de la identidad personal y social.

Este autor afirma que se debe establecer un equilibrio entre los defensores y detractores de las tecnologías y un enfoque más amplio del fenómeno. Cree que ciertos elementos, como el acusado cambio tecnológico provocado con la aparición de internet, pueden dar lugar a saltos generacionales más transcendentales que los provenientes de las usuales y predecibles discrepancias entre jóvenes y mayores que se arrastran desde tiempos remotos (Cicerón ya hablaba mal de los jóvenes de su tiempo, empleando para ello expresiones que hoy son aún comunes). No obstante, aún acordando que estos cambios en las condiciones adjuntas al progreso personal afecten al aprendizaje y al pensamiento, Buckinham indica que existe un excesivo determinismo tecnológico en aquellos que han manifestado que los jóvenes nacidos en un mundo digital tienen una forma de aprender totalmente diferente.

Nos asalta una reflexión derivada de lo anterior: ¿Quizá el cerebro humano evoluciones hacia un desarrollo mayor de las zonas relacionadas con la vista que con las de la abstracción lógica? Faltan generaciones para confirmar este extremo, pero la híper estimulación de ciertas zonas podría sugerir esta tendencia.

En este estudio de Buckinham, se toma como argumento el hecho de que las nuevas tecnologías no han aparecido en un entorno neutral sino que han sido promovidas por una mezcla de elementos económicos, sociales y políticos y que, por ello, no se puede analizar su impacto en las maneras o predilecciones de algunos colectivos sin tener en cuenta varios factores determinantes en los procesos y contextos sociales que los circunscriben. Por este motivo, considera que el determinismo digital que se encuentra bajo las fórmulas de Net Generation o nativos digitales ha ocasionado un sesgo que presenta dificultades para la comprensión de algunos 
aspectos notables: el análisis está basado principalmente en los usuarios con mayor conocimiento o facilidad de acceso a las nuevas tecnologías, estableciéndose una perspectiva enormemente maniquea sobre los contrastes entre los "antiguos" medios de comunicación (cine, radio y televisión) y los "nuevos" medios (en especial internet).

Otro de los aspectos por los cuales este estudio se presenta como una primera fase que deberá ser completada es que en él se sobrevalora la capacidad creativa y el carácter innovador de los jóvenes en los medios digitales cuando está demostrado que la mayoría se limita a buscar información y comunicarse de forma interpersonal con la gente cercana; se ha magnificado también la gran atracción que tienen los jóvenes hacia las nuevas tecnologías, extendiendo las formas de actuar de los llamados geeks o personas que sienten una gran pasión por la informática y las nuevas tecnologías a toda una generación.

Destaca además que el proceso de asimilación del aprendizaje de las habilidades sociales que se lleva a cabo en las redes y otros espacios interactivos es también relevante desde el punto de vista de la adquisición y desarrollo de destrezas cognitivas.

Si emplazamos el aprendizaje a un contexto social, debemos también concebirlo como un proceso que no es extraño a la creación de una personalidad social frente a una determinada comunidad donde se tiene, o se pretende asumir, un papel. Además, de forma análoga al proceso de socialización que permiten las redes sociales o la mensajería instantánea, los jóvenes están entrenando sus actividades cognitivas como la facilidad de recuerdo, la creación y verificación de hipótesis, la estrategia o incluso la toma de decisiones ante supuestas dificultades mediante la práctica de juegos virtuales (como los de estrategia). Ni qué decir tiene que todo esto supone un campo de nuevas teorías, pero Buckingham incide solamente en el hecho de que estos desarrollos cognitivos y sociales se realizan sin encontrarse bajo la supervisión de los adultos (padres o profesores). Es decir, aprenden a solucionar problemas concernientes con el uso cotidiano de sus computadores e internet de manera informal, a través de una metodología de ensayo-error y de colaboración con sus semejantes (incluso con programas amigos).

Por este motivo, aún admitiendo que una gran parte de los jóvenes estudiantes que han nacido con acceso a estas tecnologías está llevando a cabo todas estas diligencias con éxito, considera que debemos dejar de asombrarnos ante la disposición innata que tiene esta generación para desenvolverse con los medios y pensar en la forma en que se les puede acompañar y facilitar sus tareas, lo que en muchos casos conlleva procesos complejos. Es decir, no preocuparse por la velocidad y las formas sino por la asimilación y los contenidos. En este sentido, demanda una visión más amplia del concepto de alfabetización digital.

La alfabetización es más que una cuestión de habilidades funcionales, o saber cómo encontrar determinada información; necesitamos ser capaces de evaluar esa información si debemos transformarla en un conocimiento significativo. Esta alfabetización digital debe ser incluida en una disciplina más amplia, el estudio de los medios de comunicación. (Pisticelli 2009)

Tal y como hemos visto, las nuevas tecnologías irrumpen actualmente en las aulas sembrando en terreno ya abonado (los nativos digitales). Este hecho no puede pasar desapercibido por los 
estudios de semiótica que, utilizando las póstulas de Sausurre como cimientos sobre los que edificar, debe desarrollar una serie de nuevas herramientas lingüísticas adaptadas a las nuevas necesidades educativas en esta nueva arquitectura de comunicación simbólica y digital.

\section{Referencias}

Benveniste, E. (1977) Problemas de la lingüística general. Ciudad de México: Paidós.

Borges, J.L. (1949). El Aleph. Buenos Aires: Emecé.

Bougnoux, D.(1999) “Significar”. En: Introducción a las ciencias en la comunicación. Buenos Aires: Nueva visión.

Brea, J.L. (2007) Cultura_RAM :Mutaciones de la cultura en la era de su distribución electrónica. Barcelona: Gedisa.

Buckingham, D. (2007) Introducing Identity. Youth, Identity, and Digital Media . Cambridge: The John D. and Catherine T. MacArthur Foundation-The MIT Press. Obtenido el 26 de abril de 2010, de http: / www.mitpressjournals.org/toc/dmal/-/6

Freire, J. (30.10.2007) “¿Quiénes son los nativos digitales? ¿Y por qué?” Obtenido desde http://nomada.blogs.com/jfreire/2007/10/quines-son-los-html

Frutos, J.T. (2009) "Las organizaciones profesionales y las tecnologías de la información como nuevos valores del Periodismo". Vivat Academia n. 107. Obtenido el 22 de abril de 2010 en http://www.ucm.es/info/vivataca/numeros/n107/articulo.htm

Lotman, Y. (1999) Cultura y explosión: lo previsible y lo imprevisible en los procesos de cambio social. Barcelona: Gedisa.

Lozano, J. (1995) "La semiosfera y los estudios de la cultura” en Revista de Occidente n. 145-146. Obtenido el 4 de mayo en http://www.ucm.es/info/especulo/numero8/lozano.htm

Melero, D. (2000) “¿Estamos solos en Internet?” Vivat Academia n. 13. Obtenido el 24 de abril de 2010 en http://www2.uah.es/vivatacademia/anteriores/trece/docencia.htm

Ovelar, R.; Benito, M.; Romo, J. (2009) "Nativos digitales y aprendizaje: una aproximación a la evolución de este concepto”. Revista Icono 14 Vol. 7 n. 1 . Obtenido el 29 de abril de 2010 en www.iconol4.net

Pérez, M. (2006) "Comunicación online: Una sociedad en cambio, activa y conectada". Comunicación y Medios n. 17. Obtenido el 28 de abril de 2010 en http://www.icei.uchile.cl/comunicacionymedios/pdf/17/comunicacion_online_marcos _perez_cym_17.pdf

Piscitelli, A. (2009) Nativos Digitales: dieta cognitiva, inteligencia colectiva y arquitecturas de la participación. Buenos Aires: Santillana.

Prensky, M. (2001) "Digital natives, digital immigrants part 1". On The Horizon-The Strategic Planning Resource for Education Professionals n. 1 Vol. 6. Obtenido el 29 de abril de 2010, de http://www.ingentaconnect.com/content/mcb/274/2001/00000009/00000005/ art00001 
------- (2001) "Digital natives, digital immigrants part 2: Do they really think differently?". On The Horizon-The Strategic Planning Resource for Education Professionals n. 1 Vol. 6. Obenido el 29 de abril de 2010, en http:/ /www.ingentaconnect.com/content/mcb/274/ 2001/00000009/00000006/art00001

Sanders, Ch. (2007) La lógica considerada como Semiótica de Peirce. Madrid: Biblioteca nueva.

Saussure, F. (1994) Curso de Linguistica General. Madrid: Planeta-DeAgostini.

Scolari, C. (2008). Elementos para una teoría de la comunicación digital. Barcelona: Gedisa. (2009) Digital ECO_logies: Umberto Eco y la semiótica de los hipertextos e interfaces. Obtenido el 3 de mayo de 2010 en http://hipermediaciones.com/2009/02/25/digitaleco_logies-umberto-eco-y-la-semiotica-de-los-hipertextos-e-interfaces/

Shils, E. (1977) Sociedad de masas y su cultura: Industria cultural y sociedad de masas. Caracas: Monteávila.

Tapscott, D. (1997). Growing Up Digital: The Rise of the Net Generation. Madrid: Mcgraw-Hill. Targino, M. das G. (2008) "Presente virtual. Incertidumbre en un futuro de paz en medio de recursos tecnológicos e informacionales”. Comunicación y Medios n. 18. Obtenido el 29 de abril de 2010 en http://www.icei.uchile.cl/?_nfpb=true\&_pageLabel=conUrlICEI \&url $=52045$

Trejo Delarbre, R. (2006) Viviendo en el aleph. La sociedad de la información y sus laberintos. Barcelona: Gedisa.

Toledo Hidalgo, J. (2006) El dilema de la dimensión antropológica de la industria del entretenimiento. Ciudad de México: Universidad de Anahuac. 